2

\title{
ICTS SUPPORTING TARGETMANIA ${ }^{\mathbf{1}}$ \\ How the UK Health Sector is Trying to Modernise
}

\author{
Kathy McGrath \\ Brunel University, UK
}

\begin{abstract}
This research is concerned with a globalising discourse of modernisation, in which information and communication technologies (ICTs) are deeply implicated. It examines efforts to modernise the emergency arm of the UK National Health Service (NHS) so that it is more responsive to contemporary public demands. Pervasive in managerial and ministerial discourses about modernising the NHS are perceived joint needs to change its culture and to mobilise the potential of information technology. Drawing from critical social theory, the main argument of this paper is that the way the ambulance services are regulated and monitored is giving rise to a modernisation programme in which cultural change and ICT innovation is being narrowly conceived in terms of achieving response time targets. So in this case the process of modernisation is acting to deny autonomy locally and reinforce a 1950s image of ambulance workers that they would reject as not at all modern.
\end{abstract}

Keywords: Modernisation, information systems, critical social theory, cultural change, health sector, performance targets.

\section{INTRODUCTION}

Whether one subscribes to the view that we are now in an age of high modernity (Giddens, 1991), a postmodern era (Clegg, 1990), or that we have never been modern at all (Latour, 1993), organisations still strive to show

1 Targetmania is a neologism reflecting a popular usage of -mania as the eager pursuit of something. So targetmania indicates the eager pursuit of targets. Without presupposing that eagerly pursuing any phenomenon is essentially good or bad, this paper reveals that the government's discourse of modernising the UK ambulance services focuses attention on pursuing performance targets, and it explores what is being achieved by this pursuit 
that they are modern. In these discourses, they discuss the efficiency of their operations, the quality of service they provide, and the benefits for staff and consumers of their adoption of perceived leading edge technologies. Relative to many nations the UK is one where many such modern organisations exist, but just as modernisation programmes deriving benefits from ICTs are unevenly experienced across the world, so they are within nations. Many UK organisations still strive to be modern, and this discourse is perhaps nowhere more prevalent than within the health sector. Literature published by the Department of Health is replete with references to modernising the National Health Service (NHS) and government ministers and health service managers reinforce these messages.

This research is concerned with these modernisation efforts, in which technological rationalisation and change in UK health care organisations is aiming to make the NHS more responsive to contemporary public demands. It focuses on the ambulance services, and examines the outcomes they are achieving in efforts to exploit the potential of ICTs to improve the service they provide. The main argument of this paper is that the modernisation message for ambulance services focuses attention on meeting targets that are disjoint from the context that would give them meaning. Not only does such disjunction act to constrain local innovations that might give rise to cultural change, but also, against these homogenising measures of performance supported by ICTs, outcomes achieved indicate that parts of the service are getting worse. Following this introductory section, I present the theoretical basis of the study, followed by a description of the research setting and method used to conduct the investigation. Then I present and discuss the research findings and draw conclusions from the study.

\section{THEORETICAL PERSPECTIVE}

Pervasive in managerial and ministerial discourses about modernising the NHS are perceived joint needs to change its culture and to mobilise the potential of information technology. Although this research takes up both themes, it eschews the rationalistic perspective evident in the popular management literature that seems to inspire several government publications and ministerial speeches. In this way, the views that organisational culture can be changed in line with managerial objectives (Schein, 1985), and that particular technologies have properties that produce such effects, are seen as problematic. Attempts to change ways of organising or to implement technology to create organisational effectiveness may give rise to unintended consequences, which in turn may challenge the original objectives. 


\subsection{Power-Knowledge Relations}

This research explores the above view by adopting a Foucauldian concept of power-knowledge relations, which allows movement between a loose coupling of forms of organising and technological developments in the broader environment to an increasingly tighter coupling of the individual and technology in a specific change effort. These moves may be understood as circular movements between poles of bio-power and disciplinary power through a number of intermediate positions in which regulative methods and disciplinary techniques are fused (Foucault, 1977).

Bio-power is concerned with human life and bodies in general, in terms of how they are supervised and regulated. Issues such as 'propagation, births and mortality, the level of health, life expectancy and longevity, with all the conditions that can cause these to vary' (Foucault, 1979: p.139) are the concerns of bio-power. It is focused on specifying and controlling a whole population and therefore should be understood as having a global dimension.

Disciplinary power is a supervisory mechanism targeted at groups and individuals, and hence on their functioning and performance within an institutional setting. It targets the body by addressing issues such as its disciplining, the optimization of its capabilities, the extortion of its forces, the parallel increase of its usefulness and its docility, its integration into systems of efficient and economic controls' (ibid.). It is an individualising mechanism for subjugating bodies in a specific context through the calculated and meticulous control of time and space.

The techniques of bio-power and disciplinary power are not in opposition. Rather their evolution constituted 'two poles of development linked together by a whole intermediary cluster of relations' (ibid.). Among these linkage mechanisms, Foucault focuses on the deployment of sexuality as one of the most important arrangements in which bio-power was joined with disciplinary power. The deployment of ICTs in organisations constitutes another form in which concerns to specify and discipline bodies may be fused together.

\subsection{A Regime of Truth}

Foucault links power and knowledge in a circular relationship through discourse. He refers to this relationship as a regime of truth, arguing that:

Each society has its regime of truth, its "general politics" of truth: that is, the types of discourse which it accepts and makes function as true; the mechanisms and instances which enable one to distinguish true and false statements, the means by which each is sanctioned; the techniques and 
procedures accorded value in the acquisition of truth; the states of those who are charged with saying what counts as true. (1980: p. 131).

In this context, truth is not a fundamental, universally accepted, scientific truth but rather should be understood as knowledge that is deemed to be true by a given community. Interpretations of this knowledge emerge in social discourse and enable and constrain the courses of action available to members of the community. Action (conceived as the exercise of power) may reproduce or change the domain of knowledge, which in turn may reconfigure the power relations. Discourse, then, has three elements: a community in which the discourse emerges, institutions with knowledge and authority which delimit the discourse, and grids of intelligibility, or dispositifs, which enable particular practices to be related to each other in a specific type of discourse.

So, the way that the UK government finances, regulates, and monitors the performance of the NHS can be linked with specific change efforts within the ambulance services in which the discourse of modernisation is being enacted. Closing the circle, the disciplinary mechanisms employed to categorise particular groups within this discourse are implemented in the name of patient care and therefore these local efforts may be understood as accomplishments in which a particular service discharges its responsibility to foster the health of the nation.

\section{RESEARCH SETTING AND METHOD}

There are 35 UK ambulance services, 32 regional services in England and a single national service in each of Wales, Scotland and Northern Ireland. Although plans to modernise the NHS affect all UK services, variations apply in Scotland, Wales and Northern Ireland. This research focuses on ICT implementation and related cultural change within the 32 English services.

UK governments, past and present, have been prime movers in these developments. Through a diverse mix of consultative groups, professional advisors, surveys and opinion polls, successive governments claim to have understood contemporary demands on the health service, and they have attempted to enact various strategies for responding to these demands. In the last 20 years, we have seen moves to introduce more professional managers into the NHS (Department of Health, 1983), the establishment of an internal market (Department of Health, 1989) and, more recently, a proposal to replace the internal market with a system of integrated care (Department of Health, 1997). Each move has expressed a concern with patient care, and 
increasingly information technology has been articulated as having a central role. After 20 years of effort, then, we may ask why it is that the NHS, and specifically the ambulance service, is finding it so difficult to modernise. What characterises the discourse of modernisation and the discursive practices of ambulance workers that makes them reluctant partners?

\subsection{Ambulance Performance Standards}

For nearly 30 years, the official measure for assessing whether UK ambulance services are responding to the demands of the public has been based on how frequently they get to emergency scenes within predefined target times set at national level. Between 1974 and 1996, these performance measures, then called ORCON (Operational Research Consultancy) standards, required that:

- $50 \%$ of emergency calls should receive a response within 8 minutes, and

- $95 \%$ of emergency calls should receive a response within $14 / 19$ minutes in urban/rural areas respectively.

Only the 14/19-minute requirement was incorporated in the Patient's Charter (Department of Health, 1996), and services focused upon this target despite clinical evidence that some conditions require a faster response. In 1996, a government-initiated review of ambulance performance standards (Chapman, 1996) identified a requirement to respond to patients according to their clinical need, and hence to prioritise emergency calls so that those with the greatest need receive the fastest responses. The new ambulance performance standards were thus established. They reflect the requirements of the national performance assessment framework for coronary heart disease (Department of Health, 2000), in which the aim is to achieve a $40 \%$ reduction in deaths from heart disease and stroke in the under 75s by 2010 . Some services started to move towards the new standards in 1997 , but since March 2001 all services have had targets of responding to:

- $75 \%$ of category A (life-threatened patient) calls within 8 minutes, and

- $95 \%$ of categories B (serious, not life-threatened) and C (neither serious nor life-threatened) calls within 14/19 minutes in urban/rural areas.

Both the 8-minute and the 14/19-minute standards are included in Your Guide to the NHS (Department of Health, 2001), the current replacement for the Patient's Charter, but early evidence shows that the 8-minute standard is now the focus of attention (Department of Health web site, 2002). 


\subsection{Computerisation Within the Ambulance Services}

Ambulance services have adopted particular types of ICTs in their drive to enhance performance. From the late 1970 s to the mid-1990s, performance to ORCON standards became an increasing focus of attention, especially when the requirements of the internal market in the NHS started to emerge in 1989. In line with these standards, ICTs addressed speed of response assuming all calls had equal priority. During this period, these developments took the form of implementing computer aided dispatch (CAD) systems.

CAD systems were concerned with taking and responding to emergency calls more quickly based on up to date information about the position and status of suitable resources. However, by incorporating monitoring mechanisms, in particular tracking the locations and status of resources, they challenged the task orientation of vehicle crews, who previously had relative freedom to manage the cycle time involved from receiving an emergency call to notifying availability to attend another incident. Typically this cycle may be from 50 to 80 minutes, and includes the times to get to the incident, to assess the patient at the scene, to convey the patient to hospital, to comply with hospital procedures regarding handover of the patient, and to notify availability following patient handover. Both ORCON standards and the new ambulance performance standards address the first task in this cycle only.

The move, in the late $1990 \mathrm{~s}$, to the new ambulance performance standards changed the basis on which emergency responses are provided and service performance is measured. At this time, services modified their CAD systems to embed within them applications referred to as priority dispatch (PDS) systems. Through a structured series of question and answer protocols and embedded rules, PDS systems guide call takers through a process that suggests a clinical determinant indicating the severity of a patient's condition. Thus they enable the priority of each call to be assessed so that vehicles can be dispatched to patients according to the priorities assigned.

Although the determinant attached to each medical condition is determined at organisational level, ambulance services have limited freedom to decide on the category of response provided for each complaint. Indeed, in some cases the priority category is mandated at national level, as in the government imperative that suspected cardiac arrests receive a category $\mathrm{A}$ response (Department of Health, 2000). In this way, a deviation from the discipline can have serious consequences for the ambulance service and the control room staff concerned, who will be accountable for the outcomes of such action. This eventuality may be seen as giving rise to a situation in which the suggested determinant is accepted uncritically, rather than risk the consequences of overriding it. 


\subsection{Research Method}

The data for this research were drawn from three main sources: literature produced by the Department of Health, including statistics about ambulance service performance; seminars and exhibitions held at the UK national conference for ambulance services (Ambex) in July 2000; and my empirical work with a UK ambulance service. The literature outlines the government's strategy for modernising the NHS, the Ambex conference addresses the way that ambulance services are responding to it, and my fieldwork gave me some detailed insights on the type of initiatives at the heart of these responses. At the conference, I heard presentations from the Health Minister, several ambulance service senior managers and chief executives, and a number of researchers working in the field. I also saw demonstrations of the ICTs and other resources being used by ambulance services to address the modernisation programme. My fieldwork with one service involved a longitudinal study of 1-2 days per week for 12 months during which time I observed a change programme to implement the priority dispatch initiative. I use the theoretical framework outlined earlier to analyse these efforts, and specifically to address the research question posed at the start of this section about how the discourse of modernisation challenges existing practices.

\section{CASE ANALYSIS}

The ambulance services are a community in the sense that acting as providers of pre-hospital patient care grounds the identities of each of them. All are subject to the same government monitoring mechanisms and each has access to public funds and other resources relative, in principle, to the demands of the population it cares for. Moreover, as part of responding to the needs and expectations of those they serve, each has adopted particular types of ICTs (perceived as performance enhancing) called CAD systems and PDS systems. In Foucault's (1980) sense, then, these mechanisms are the basis of a regime of truth for the ambulance service community, and modernisation in line with government imperatives is a discourse to which they are being applied.

Bio-power circulates in the arrangement in which government through the various arms of the NHS addresses the health needs of the population. The funding bodies, patients' charters, ambulance performance standards, and other regulatory methods constitute grids of intelligibility, which aim to address patient care by matching resources to demand in systems of categorisation, measurement, and normalisation. The specific aim to reduce premature deaths from coronary heart disease is an example of particular 
importance. This goal involves a regulatory mechanism with four main aspects. Calls to the ambulance services are categorised based on clinical need, targets are set for emergency response times to each category of call, the performance of the services against target is continually measured, and funding for subsequent periods of operation is based on an evaluation of previous performance. The norms of performance for ambulance services, effective since March 2001, are enshrined in the new ambulance performance standards, and the implementation of information systems was seen as 'essential to the proposed changes' (Chapman, 1996: p. 6).

Disciplinary power operates at the level of individuals and groups. The implementation of local PDS systems introduces a disciplinary mechanism within working practices designed to optimise staff members' individual performances with the aim of improving the overall performance of the service. It aims to standardise the process of call taking so that the priority assigned to each call is a function of the call taker's compliance with the discipline rather than a function of his or her individual human judgement of the clinical priorities. Furthermore, by limiting the dialogue with the caller to a predetermined exchange of questions, answers and instructions, it aims to provide a response to the patient in the fastest time possible.

Moreover, local quality assurance units have been introduced within ambulance services to monitor compliance with the new discipline. These units evaluate a small percentage (typically 1-3\%) of the emergency calls received each month. Call takers receive an evaluation record and compliance rating which feeds into their periodic assessments by managers. Thus call takers are subjected to a disciplining mechanism and may be subjectified by it in the sense that, by reflecting on their evaluations and addressing the issues raised, they participate in their own monitoring.

So the implementation of PDS systems embedded within CAD systems, which in combination are inscribed with the values of the new ambulance performance standards, attempts to fuse together the government's plans for modernising the NHS and the ambulance services responses to them. However, there are some contradictions within this regime of truth and some unintended outcomes. For example, members of the ambulance services note that framing the performance targets in terms of response times means that:

If you get there in 8 minutes and the patient dies you've succeeded, but if you get there in 9 minutes and the patient lives you've failed (Director of Corporate Resources, Ambulance Service, quoted in Nicholl et al. (2001: p.16). 
In this way, many argue:

They are not real targets. The real targets are the ones that have clinical outcomes (Priority dispatch manager, Ambulance Service)

The view that clinical outcomes are the real measure of success is an increasingly pervasive discourse within the ambulance service community. The new ambulance performance standards may be seen as medium for and an outcome of this discourse. On the one hand, the new standards emphasise the need to provide an 8-minute response to life-threatened cases, which the previous standards did not; so current assessments of service performance take into account what the outcomes for patients might be. On the other hand, the outcomes achieved (for example, the number of patients that leave hospital alive following out-of-hospital cardiac arrest) still do not form part of the performance measures for ambulance services. Indeed, hospitals do not pass information to ambulance services about the clinical outcomes achieved by emergency patients, so that ambulance performance figures are de-rooted from the context that would give them meaning. Moreover, such one-way traffic sustains the 1950s image of ambulance workers as 'glorified porters' (Radio 4, 2002a), and reinforces the sense among them that they occupy a marginal position in NHS plans (Ambex, 2000).

\subsection{A Discourse with the Health Minister}

At the Ambex conference in July 2000, the Health Minister advised the ambulance services of what they need to do to modernise:

We need to change the way the NHS works ... status quo is unacceptable ... public expectations have changed ... technology has advanced ... what we are about is treatment based on clinical need... We are not trying to create league tables ... however we do need to learn from each other. ... In the past there may have been a culture where one trust competed with another ... but as far as this government is concerned, this is not the way. ... We need to teach the public to help themselves ... to make the service the same wherever you are ... to work together. ... Targets need to be met.

At this time, a number of ambulance services had already installed a PDS system, and several of them were already getting close to or regularly meeting the new standards. Indeed, the Minister said 'I cannot but pick out Staffordshire Ambulance Service' in this regard, while declaring that the services yet to achieve their targets would be expected to show considerable progress by 
March 2001. The chairman of the London Ambulance Service asked the Minister how much she believed could be achieved by the new practice of triaging (prioritising emergency calls) over the telephone, by comparison with the former practice of triaging when the patient was examined. She suggested that 'there are a number of days you get through on a wing and a prayer', but she held fast to the aims of telephone triage so that resources could be dispatched according to patient need - a need assessed using a PDS system. Still, members of the ambulance services are concerned about the efficacy of the new regime because they feel that without a medical examination much uncertainty is involved in assessing the urgency of clinical need. Indeed, they cite cases where prioritisation at the point of call receipt does not achieve the desired outcome - that those in the greatest need receive the fastest responses.

For example, when a member of the public calls the ambulance service to report that someone has collapsed in the street, the caller's responses to standard questions may result in an automated assessment that the patient is life-threatened and needs a category A 8-minute response. Yet when a caller describes the condition of a person who, say, has fallen off a building site, the assessment may indicate a serious and very painful back injury, but not one that is life-threatening, hence a category B 14/19-minute response. However, when ambulance staff arrive on the scene, they may assess the first patient as a substance abuser, who lost consciousness under the influence of a substance and does not require an emergency response at all. The second patient invariably will be conveyed to hospital, hence had the greater clinical need, but the seriousness of these conditions could not be gauged until the patients were examined. So there is a concern that the ICTs in use for call prioritisation are not yet sophisticated enough to pick out reliably and safely calls requiring different levels of response (Nicholl et al., 2001).

Responding to a question from a senior executive at the East Anglian Ambulance Service, the Minister said:

Any notion that we are prepared to lower the standards - no.

This exchange reflects concerns within the ambulance services about the demands of meeting 'a far tougher standard' (IT director, Ambulance Service). In effect, priority dispatch requires that fewer calls receive an 8-minute response than did ORCON, but the new standard is perceived as more demanding because ORCON allowed any $50 \%$ of calls reached in 8 minutes to be counted. In this way, the ORCON regime created pressure to dispatch resources as quickly as possible to each call regardless of how urgent it seemed, while deemphasising the implications of having no resources available when, say, a "suspended" patient - one who has stopped breathing - was reported. Under a priority dispatch regime, services must reach 75\% (during 2003/04, $90 \%$ ) of category A calls in 8 minutes. These calls may be only $20-30 \%$ of their work, but they occur all of the time. In this way, services have to manage their 
resources much more dynamically than before to ensure that their vehicle responses match a changing profile of call priorities in the control room. In the above scenario, this would mean diverting an ambulance on its way to a lower priority call to respond to the "suspended" patient.

A further comment from the Ambex 2000 conference is interesting in this regard. The chief executive of Staffordshire Ambulance Service argued that staff should dispatch an ambulance as quickly as possible and worry about the priority later - when the patient is examined. Delegates were well aware that Staffordshire had been responding to over $80 \%$ of all calls within 8 minutes for some time, and therefore had not only been exceeding the new 8-minute target before it took effect, but exceeding the then current ORCON 8-minute target by over $60 \%$. Nevertheless, none of the other trust board directors suggested they were sanguine enough to attempt a deferred prioritisation strategy in their services, lest in delaying triage they failed to achieve a sufficiently high number of 8-minute responses to be sure of including the required number of lifethreatened cases. Indeed, the chief executive of the Essex service, an advisor to the Department of Health, argued that prioritisation is essential when the call is received, especially for the larger services like Essex and London - the latter has about 9 times the call volume of Staffordshire. Still, it is interesting to note that the service that is out-performing all others, including the $50 \%$ that are smaller than it, is one that has chosen not to dispatch by priority.

\subsection{Outcomes Achieved}

The following table summarises the latest available (Department of Health web site, 2002) yearly performance figures for ambulance services.

Table 1. Summary of ambulance service performance figures 1999-2002

\begin{tabular}{lrrr}
\hline & $99 /$ & $00 /$ & $01 /$ \\
& 00 & 01 & 02 \\
\hline & 20 & 31 & 32 \\
a. No of services operating to the new performance standards & 1 & 3 & 14 \\
b. No of (a) achieving the category A 8-minute standard & 12 & 13 & 16 \\
c. No of (a) achieving the category B/C 14/19-minute standard & $*$ & 1 & 1 \\
d. No of (a) where cat. A performance dropped from previous year & $*$ & 0 & 0 \\
e. No of (d) where performance dropped more than 5 percentage points & $*$ & $*$ & 0 \\
f. No of (d) where cat. A performance dropped in 2 successive years & $*$ & 11 & 3 \\
g. No of (a) where cat. B/C performance dropped from previous year & $*$ & 0 & 3 \\
h. No of (g) where performance dropped more than 5 percentage points & $*$ & $*$ & 3 \\
i. No of (g) where cat. B/C performance dropped in 2 successive years & $*$ & & \\
& & & \\
\hline
\end{tabular}


Results in Table 1 show a mixed performance against government targets. Against the category A target, services tend to improves their performance year on year, an increasing number are reaching the $75 \%$ target, nevertheless in 2001/02 only 14 (of 32) achieved it. Category $\mathrm{B} / \mathrm{C}$ responses are more variable. Performance fluctuates year on year, with $40 \%$ of services showing a drop in performance in 2001/02, and $10 \%$ of cases showing a significant drop of more than 5 percentage points. Moreover, $10 \%$ of services have a declining category $\mathrm{B} / \mathrm{C}$ performance over 2 years, and only $50 \%$ of services are yet achieving the $95 \%$ target. The current trend, then, seems to be for ambulance services to focus resources on meeting the 8minute standard, with the result that category $\mathrm{B}$ and $\mathrm{C}$ patients in some areas are experiencing a less prompt service. Clearly, one compelling issue is how this performance is affecting clinical outcomes, but while hospitals and ambulance services continue to report their performance separately there is no way of comparing patient outcomes according to wait times for an ambulance. However, one can question the extent to which comparing ambulance services using the current standards of performance gives rise to meaningful results.

Although the national framework for coronary heart disease (Department of Health, 2000) requires that suspected cardiac arrests receive a category A response, not all patient complaints are specified as clearly. Services have some scope to decide how they allocate other complaints to the categories A, $\mathrm{B}$ and $\mathrm{C}$, which affects the response targets they must meet. Moreover, services vary in the degree of accuracy with which their systems record response times to calls. For example, some services require ambulance crews to press a button in their cabs when they arrive at an emergency scene so that their computer systems can record the time precisely. Others require crews to fill in a paper form, which may not be completed until patient handover at hospital up to one hour later, when the arrival time may not be remembered so precisely. When the clock starts ticking is also variable. A Department of Health (1998) directive states that this should be when the caller's telephone number, the incident location and the chief complaint have been logged by the call taker, but not all services time this process so precisely. Moreover, some services use automated Caller Line Identity (CLI) to identify the caller's address, which may be the incident location, so the time between call connection and the start of timing is variable, and this time is not reflected in current performance figures even though patient outcomes depend on it.

So efforts 'to make the service the same wherever you are' is a discourse of an ideal type, the more so when we consider how the clinical skills of ambulance crews may vary, and how geographical area affects the time needed to make an emergency journey. This argument is not to suggest that we should accept a postcode or some other form of "lottery" for the 
ambulance service, but that a focus on national standards may be stifling local innovation and change which might find other ways of improving the service. Indeed, the history of the microcomputer, the Internet, and several strategic information systems has shown us how local innovations can radically change the way that people and communities work.

\section{DISCUSSION}

This research has focused on how the UK ambulance services are trying to modernise. Although themes of cultural change and ICT innovation are articulated in the messages from health service managers and government ministers, they are still firmly rooted in an instrumental discourse that sees these processes linked directly with organisational performance. Moreover, that performance is measured in terms of a narrow response time target that seems meaningless to many required achieving it. So the government's rationale for modernising the NHS reinforces an identity of ambulance workers that they reject - that their value should be measured by their ability to provide rapid transport rather than contribute to patient outcomes.

Within the ambulance service that was the focus of my fieldwork effort, staff respond to current modernisation initiatives in ways that reflect a number of the concerns raised at the Ambex conference. Questioning the efficacy of a computerised call prioritisation regime, some control room staff will bypass or override certain PDS protocols, drawing upon their own knowledge and experience to assess the clinical priorities. Most, though, will not risk this course of action even when they feel a need for it, lest they find themselves accountable to a coroner's court and subject to disciplinary action. Many ambulance crews will respond especially rapidly to calls that they judge may provide an opportunity to demonstrate their clinical skills, irrespective of the priority assigned by control room staff using the PDS system. In this way, a road traffic accident will usually receive a very rapid response, even when information from the caller suggests there are only minor injuries because the accident happened at very low speed in congested traffic conditions. So the working practices of staff indicate a desire to contribute to clinical outcomes, a recognition of the need for prioritised responses, but a lack of trust in PDS systems and a challenge to the way that being responsive is conceived within the new performance standards.

So, although recent moves within the ambulance services endeavour to differentiate between categories of patient so that those in the greatest need get the fastest responses, three major problems with targets are evident in these developments. First, the emphasis in the new standards on providing the most rapid responses to category $\mathrm{A}$ cases means that this target is 
focused upon at the expense of category B and C cases, some of whom now receive a slower and hence (on a response time measure) a worse service. Second, national targets deny autonomy locally. Only one service claims to reject the telephone triage message, but Staffordshire achieved autonomy by exceeding the targets without accepting the message. Surely we should now ask if there are ways of improving the service without focusing narrowly on response time targets. Third, target outcomes are constructed. Ambulance services time their responses in different ways, some of which are more open to construction than others. Moreover, when scrutiny is intense and targets seem meaningless how the construction is achieved may be open to abuse. Indeed, in a recent poll of health service managers (Radio 4, 2002b), 1 in 12 admitted to 'massaging their figures'. Although this poll was not specifically targeted at ambulance services, it reveals that the truths NHS performance figures purport to tell are not accepted by all within the service, far less that they constitute an objective truth that all of us should accept.

\section{CONCLUSION}

Modernisation efforts may be accepted because they embody desirable goals - to be seen as abreast of the latest developments in a field, as adopting contemporary technologies, and as being responsive to the world in which we live. Alternatively, they may challenge existing ways of organising and established ideas of providing a service. Both such manifestations are evident in current modernisation programmes within the UK NHS, but the difficulties with implementing these programmes does not reduce to a straight conflict between those advocating new ways of being responsive and those wishing to retain existing ways. Being modern is narrowly conceived in terms of performance targets so that innovation and change means getting faster. Moreover, since targets are framed by response times they are disjoint from the patient outcome context they are alleged to serve. The in-order-to aspect of modernisation is being addressed, but a link with the because-of motives (Ciborra, 1999) that would give it meaning has not been established.

Modernisation is a globalising discourse in which the use of ICTs is deeply implicated. However, in the UK ambulance services those advocating modernisation and those who need to accept and enact it are at most loosely aligned and the way that ICTs are being adopted creates limited scope to fuse these interests together. Perhaps, though, a different type of fusion should be attempted. Rather than just trying to make conversations with callers so fine-grained that a patient's condition can be determined over the telephone, the potential of ICTs to connect different communities should be exploited. If ambulance services knew more from hospitals about the 
outcomes achieved by their patients, the need for innovation and change might seem more compelling and then ICTs would achieve more than supporting targetmania.

\section{REFERENCES}

Ambex. Ambulance Services Association UK National Conference, Harrogate, UK, 1-3 July 2000.

Chapman, R. Review of Ambulance Performance Standards, Department of Health, UK, July 1996.

Ciborra, C. "A theory of Information Systems Based on Improvisation", in Rethinking Management Information Systems, W. Currie and R. Galliers (eds.), Oxford: Oxford University Press, 1999.

Clegg, S. Modern Organizations: Organization Studies in the Postmodern World, London: Sage, 1990.

Department of Health. "Report of the NHS Management Inquiry", Department of Health and Social Security, London, 1983.

Department of Health. Working for Patients, London: HMSO, 1989.

Department of Health. The Patient's Charter, London, November 1996.

Department of Health. The New NHS: Modern, Dependable, London: HMSO, December 1997.

Department of Health. "Measuring When the Clock Starts and Stops", MISC (98:23), London, 1998.

Department of Health. "Modernisation of Ambulance Services", HSC 1999/091, London, April 1999.

Department of Health. "National Service Framework for Coronary Heart Disease", HSC 2000/012, London, April 2000.

Department of Health. Your Guide to the NHS, London, September 2001.

Department of Health Web Site. http://www.doh.gov.uk/public/sb0213.htm. Statistics Division entries, accessed 7 October 2002.

Foucault, M. Discipline and Punish, Harmondsworth: Penguin, 1977.

Foucault, M. The History of Sexuality, Volume 1: An Introduction, Harmondsworth: Penguin, 1979.

Foucault, M. Power/Knowledge: Selected Interviews and Other Writings 1972-1977, C. Gordon (ed.), Brighton, Sussex: Harvester Press, 1980.

Giddens, A. Modernity and Self-Identity, Cambridge: Polity Press, 1991.

Latour, B. We Have Never Been Modern, Harvester Wheatsheaf, Hemel Hempstead, 1993.

Nicholl, J., Turner, J. and Martin, D. The Future of Ambulance Services in the United Kingdom: A Strategic Review of Options for the Future of Ambulance Services, London, Ambulance Service Association, 2001.

Radio 4. "Emergency", July 15, 2002a.

Radio 4. "Today Programme”, October 7, 2002b.

Schein, E. Organizational Culture and Leadership, San Francisco: Jossey-Bass, 1985. 


\begin{abstract}
About the Author
Kathy McGrath is a lecturer in Information Systems at Brunel University in the UK. She was born and educated in England, and holds an $\mathrm{MSc}$ and $\mathrm{PhD}$ in Information Systems from the London School of Economics. Her research focuses on the development, management and use of ICTs, and their organisational and social consequences. Kathy can be reached by e-mail at Kathy.McGrath@brunel.ac.uk.
\end{abstract}

